\title{
La question du mal chez Hannah Arendt : rupture ou continuité?
}

\author{
Sophie Cloutier
}

En 1945, Hannah Arendt écrivait : «the problem of evil will be the fundamental question of post-war intellectual life in Europe » (Arendt, Essays in Understanding 134). Bien que peu d'intellectuels se soient effectivement penchés sur cette question, elle est devenue un thème majeur de la pensée arendtienne, un questionnement qui demeure aussi, chez elle, toujours lié à l'événement politique. La préoccupation pour cette problématique a surgi à la suite de la découverte de l'horreur des camps d'extermination, dont Arendt prit connaissance en 1943. Auschwitz fut un événement bouleversant, un abîme qui s'ouvrait devant elle et qui agit comme catalyseur de sa pensée. Auschwitz se révéla la figure extrême du mal politique, un événement avec lequel Arendt ne pouvait se réconcilier, mais qu'elle tenta tout de même de comprendre. Cette analyse se retrouve dans Les origines du totalitarisme où elle utilise l'expression kantienne de «mal radical». En 1961, Arendt vécut un autre événement perturbant lorsqu'elle assista, à titre d'envoyée spéciale du New Yorker, au procès à Jérusalem du criminel nazi Adolf Eichmann. Avec la vive controverse qui suivit la réception de l'ouvrage Eichmann à Jérusalem et qui l'ébranla énormément, Arendt fut amenée à forger un nouveau concept, celui de la «banalité du mal».

Nous voilà donc face à deux concepts qui tentent de cerner la nature du mal : celui de «mal radical » et celui de «banalité du mal». Y a-t-il continuité ou rupture entre ces concepts? 
À première vue, il semble y avoir un changement de perspective, mais ce changement instaure-til réellement une rupture dans l'œuvre arendtienne? Richard J. Bernstein, dans Hannah Arendt and the Jewish Question, affirme qu'un même cheminement de pensée a conduit Arendt de la notion de «mal radical » à celle de «banalité du mal». Nous suivrons ainsi, dans un premier temps, la thèse de Bernstein sur la continuité dans l'œuvre d'Arendt, à travers l'enquête de Bernstein sur le sens de l'expression «mal radical». Nous nous pencherons ensuite sur la «banalité du mal» propre à Eichmann. Dans un troisième temps, nous proposerons d'approfondir la thèse de Bernstein en analysant la distance arendtienne face à la Critique de la raison pratique de Kant. Pour ce faire, il nous faudra nous pencher sur La vie de l'esprit et sur les cours que donna Arendt sur Kant. Nous verrons ainsi que non seulement il y a une continuité possible entre le «mal radical» et la «banalité du mal», mais qu'il importe de conserver et d'articuler les deux notions afin de comprendre l'ampleur du phénomène totalitaire. Cependant, afin de rendre possible cette articulation, la notion de «mal radical» doit être épurée de sa connotation kantienne. Enfin, nous proposerons de considérer l'importance de la pensée de saint Augustin dans la réflexion arendtienne sur le mal. La compréhension augustinienne du mal comme privatio boni et la conception augustinienne de la volonté nous permettront d'apporter un éclairage nouveau sur la notion de «banalité du mal ». Notre lecture sera donc axée sur le rôle de la volonté dans la question du mal, un aspect moins étudié par les commentateurs d'Arendt que celui du rôle de la pensée ou du jugement ${ }^{1}$. 


\section{Le totalitarisme ou le mal radical}

Dans Les origines du totalitarisme ${ }^{2}$, Arendt a tenté de comprendre ce qui pu se produire pour que l'Allemagne en arrive à un système de domination totale. Bien que le totalitarisme soit un événement sans précédent, ses germes proviennent de notre modernité ${ }^{3}$ et de son incapacité à résoudre le problème du vivre-ensemble, un problème attesté par l'antisémitisme et l'impérialisme. Le fil qui relie les trois parties de l'ouvrage, soit Sur l'antisémitisme, L'impérialisme et Le système totalitaire, est la «marche descendante vers la "désolation" » (Enegrén 208) et l'apparition du mal politique, c'est-à-dire la superfluité des hommes ${ }^{4}$.

Dans le chapitre «From Radical Evil to the Banality of Evil » de son ouvrage Hannah Arendt and the Jewish Question, Richard J. Bernstein s'emploie à retracer l'apparition et la signification de l'expression «mal radical » dans l'œuvre arendtienne. La première apparition qu'il note de l'expression «mal absolu » se trouve dans l'article «The Concentration Camps », paru en 1948 dans le Partisan Review, qui sera revu pour être inclus dans Les origines $d u$ totalitarisme. Arendt liait le «mal absolu » à la question du «tout ou rien » :

The fear of the absolute Evil which permits of no escape knows that this is the end of dialectical evolutions and developments. It knows that modern politics revolves around a question which, strictly speaking, should never enter into politics, the question of all or nothing : of all, that is, a human society rich with infinite possibilities; or exactly nothing, that is, the end of mankind. (748)

Dans la première édition des Origins of Totalitarianism, Arendt avait inclus des éléments de cet article dans ses «Concluding Remarks ». Elle parlait encore de «mal absolu», mais le liait dès lors à la question de la superfluité : «Difficult as it is to conceive of an absolute evil even in the face of its factual existence, it seems to be closely connected with the invention of a system in which all men are equally superfluous » (Arendt, Origins of Totalitarianism [1951] 433; cité par Bernstein, Hannah Arendt 140). Ce n'est qu'après la révision de ces «Concluding 
Remarks » pour l'édition de 1958, qu'Arendt ajoute l'expression de «mal radical » et un commentaire sur Kant. Ce commentaire est très important pour la suite de notre propos. En effet, Arendt regrette, en quelque sorte, que Kant, tout en pressentant l'existence de ce mal, l'ait immédiatement «rationalisé ». Elle écrit dans Le système totalitaire : «Kant, le seul philosophe qui, dans l'expression qu'il forgea à cet effet, dut avoir au moins soupçonné l'existence d'un tel mal quand bien même il s'empressa de le rationaliser par le concept "d'une volonté perverse", explicable à partir de mobiles intelligibles »(201). Nous pouvons déjà pressentir ce qui sera problématique dans la conception kantienne du mal radical, à savoir la question de la volonté et des motifs - ce qu'Arendt décrivait comme une « rationalisation ». Avant de poursuivre cette question, il importe de revenir sur l'interprétation de Bernstein.

Bernstein attire l'attention du lecteur sur une dernière «pièce à conviction » dans son enquête sur la signification de l'expression «mal radical ». Il s'agit d'une lettre qu'Arendt avait écrite à Karl Jaspers, datée du 4 mars 1951, dans laquelle elle s'expliquait sur sa compréhension du mal totalitaire. Elle confiait à Jaspers que la religion ne peut plus aider à comprendre le mal politique moderne, soulignant que le Décalogue n'avait pu prévoir les crimes récents. En fait, Arendt doutait même que la tradition occidentale puisse les comprendre puisqu'elle souffre d'un préjugé : «Western Tradition is suffering from the preconception that the most evil things human beings can do arise from the vice of selfishness. Yet we know that the greatest evils or radical evil has nothing to do anymore with such humanly understandable, sinful motives » (ArendtJaspers, Correspondence 166) ${ }^{5}$. Bernstein n'insiste pas sur ce passage de la lettre, mais sur ce qui vient immédiatement après, c'est-à-dire la description qu'Arendt donne de ce mal. Elle expliquait qu'elle ne savait pas exactement ce qu'était ce mal radical, mais qu'il semblait lié au phénomène suivant : 
making human beings as human beings superfluous (not using them as means to an end, which leaves their essence as human untouched and impinges only on their human dignity; rather, making them superfluous as human beings). This happens as soon as all unpredictability - which, in human beings, is the equivalent of spontaneity - is eliminated. (Correspondence 166)

Elle poursuivait sa lettre en déplorant l'illusion d'omnipotence propre à l'homme qui avait présidé à l'apparition du mal radical : si un individu singulier se croit omnipotent, il rend du même coup tous les autres individus superflus et nie la pluralité. Voilà qui préfigure la critique qu'elle fit de la subjectivité moderne et du fantasme de l'absolue indépendance du moi-voulant, lors de sa discussion sur la volonté dans La vie de l'esprit.

Bernstein insiste à juste titre sur le fait qu'Arendt ne mentionne à aucun moment que le mal radical serait lié à la nature monstrueuse des hommes. Arendt rejetait une telle conception avant même d'avoir assisté au procès d'Eichmann (Hannah Arendt 142). L'affirmation que le mal radical ne s'explique pas par des intentions mauvaises ou par des motifs méchants (evil motives) place Arendt en rupture avec la tradition occidentale de la philosophie morale. Nous pouvons même demander si Arendt est vraiment en position de rupture avec la tradition ou si elle ne fait qu'attester de la rupture morale moderne, c'est-à-dire de l'impuissance de notre philosophie morale à comprendre le mal politique. Dans son cours Some Questions of Moral Philosophy, elle parlait justement de l'effondrement des valeurs, «the total collapse of all established moral standards in public and private life » (Arendt, Responsability and Judgment 52). Quoiqu'il en soit, l'affirmation provocante que le mal ne s'explique pas par un retour sur l'intentionnalité est tout à fait cohérente avec ce qu'elle écrivait sur Eichmann, c'est-à-dire qu'«on ne parvient pas à découvrir en Eichmann la moindre profondeur diabolique ou démoniaque » (Arendt, Eichmann 460). D'autant plus que dans Le système totalitaire, Arendt n'utilise pas l'expression de «mal radical» pour qualifier le caractère psychologique des 
individus, mais pour faire une description phénoménologique du monde totalitaire ou, plus précisément, une description de la dissolution du monde commun. L'épithète « radical » vient illustrer le caractère total d'un mal qui vise une transformation de la nature humaine, voire même une éradication de l'humanité de l'homme. Comme elle l'écrivait dans Le système totalitaire, «[1]e dessein des idéologies totalitaires n'est donc pas de transformer le monde extérieur, ni d'opérer une transmutation révolutionnaire de la société, mais de transformer la nature humaine elle-même » $(200)^{6}$.

L'analyse arendtienne du totalitarisme est tout entière centrée sur la destruction du monde commun, la disparition de la pluralité et l'élimination de la spontanéité humaine. Elle distingue donc ce type de régime de la tyrannie, puisque la tyrannie autorise encore l'action, même s'il s'agit d'une action motivée par la peur : l'isolement de la tyrannie n'atteint pas la sphère privée des gens. Dans le totalitarisme, «le cercle de fer de la terreur totale ne laisse pas d'espace à une telle vie privée et [...] l'auto-contrainte de la logique totalitaire détruit chez l'homme la faculté d'expérimenter et de penser aussi certainement que celle d'agir » (Le système totalitaire 225). Ce qui était qualifié d'isolement dans la tyrannie, devient la désolation dans le régime totalitaire. Dans Le système totalitaire, Arendt écrit donc que la domination totalitaire «se fonde sur la désolation, sur l'expérience d'absolue non-appartenance au monde, qui est l'une des expériences les plus radicales et les plus désespérées de l'homme » (226). Cette épreuve de la désolation est vécue comme l'effet d'une violence qui se diffuse de l'intérieur même du corps social. Afin d'en arriver à ce stade ultime de désolation, le totalitarisme a besoin des conditions d'une masse d'individus atomisés, c'est-à-dire qu'il doit briser le tissu social pour que les individus perdent leur point de référence normalement garanti par leur position sociale ${ }^{7}$ 
La source du mal radical se trouve ainsi dans l'expérience de la désolation, dans la perte de l'espace entre les hommes, qui est nécessaire à la création d'un monde commun et aussi de toute vie politique. Dans une très belle formule, Miguel Abensour résume la conception arendtienne du monde commun : «Le monde naît de l'espace entre les hommes, de l'espace intermédiaire entre les hommes qui habitent la terre au pluriel. Espace qui fait lien et sépare tout à la fois, mieux, espace qui relie parce que d'abord il sépare » (Abensour 202). C'est à cet « exister au pluriel » que le totalitarisme s'attaque : il élimine toutes possibilités de vie avec les autres. Pour saisir le caractère radical de l'expérience de désolation, il faut la distinguer de l'isolement et de la solitude.

L’isolement correspond au fait d'être privé du domaine politique, d'être confiné à la sphère privée, par exemple. L'isolement touche ainsi la capacité d'agir avec les autres et rend donc politiquement impuissant — le pouvoir, selon Arendt, émergeant lorsque les hommes agissent de concert. La solitude se rapporte au mode d'être de la pensée. Pour penser, l'individu doit se retirer du monde ${ }^{8}$, mais à ce moment il n'est jamais absolument seul. En effet, le penseur est toujours seul avec lui-même : sa pensée exprime un dialogue silencieux entre lui et lui-même. Face à ce schéma, la désolation est la pire expérience de toutes, puisqu'elle atteint la vie humaine dans son tout, aussi bien la vie privée que la vie publique. La personne se retrouve alors entourée de gens avec qui il lui est impossible d'entrer en contact et qui peuvent même lui être hostiles. Dans cette expérience radicale, la personne désolée n'arrive plus à avoir accès à sa propre solitude : son moi l'abandonne.

Il est à noter qu'Arendt souligne qu'Épictète est le premier philosophe à avoir distingué entre solitude et désolation. Dans Le système totalitaire, elle écrit justement : «Sa découverte était, en un sens, accidentelle, sa préoccupation majeure n'était ni la solitude, ni la désolation, 
mais l'être seul (monos) au sens d'une absolue indépendance»(226). Arendt reviendra sur Épictète à la fin de sa vie, lors de son enquête sur la faculté de vouloir. Épictète tient alors le rôle du philosophe de la vie intérieure qui a proclamé la «toute-puissance de la volonté », ce qui n'est pas sans rappeler la volonté du Führer. Cependant, si la volonté gouverne la vie de l'esprit, l'esprit perd son harmonie et est continuellement en conflit. Il risque alors de perdre la capacité d'entretenir un dialogue silencieux. Comme l'explique Arendt dans Le vouloir, la toutepuissance de la volonté a un prix élevé pour la vie de l'esprit : «la pire chose qui, du point de vue du moi pensant, puisse arriver au deux-en-un, à savoir "être en désaccord avec soi-même", est devenue inséparable de la condition humaine » (102). Nous approfondirons cet aspect dans la troisième section de cet article : en effet, le changement de perspective entre le «mal radical » et la «banalité du mal » doit être lu dans l'optique de l'enquête arendtienne sur la vie de l'esprit, et notamment de sa discussion sur le vouloir. Ce qu'il faut toutefois saisir pour l'instant, c'est que la désolation s'immisce jusque dans l'amitié que le moi doit entretenir avec lui-même afin de penser. Il faut voir de plus que la pluralité essentielle au monde est aussi une condition essentielle à la vie de l'esprit elle-même. Un extrait du Système totalitaire permet de voir qu'Arendt anticipe son travail ultérieur sur Eichmann à Jérusalem et sur La vie de l'esprit :

Ce qui rend la désolation si intolérable, c'est la perte du moi, qui, s'il peut prendre réalité dans la solitude, ne peut toutefois être confirmé dans son identité que par la présence confiante et digne de foi de mes égaux. Dans cette situation, l'homme perd la foi qu'il a en lui-même comme partenaire de ses pensées et cette élémentaire confiance dans le monde, nécessaire à toute expérience. Le moi et le monde, la faculté de penser et d'éprouver sont perdus en même temps. (229)

En approfondissant cette réflexion sur le phénomène totalitaire et à la suite du procès d'Eichmann, Arendt en arrive à la notion de vacuité de la pensée et au concept de «banalité du mal ». Dans une lettre datée du 23 juin $1963^{9}$, Gershom Scholem demandait à Arendt de 
s'expliquer sur ce changement de perspective. Ne comprenant pas ce qu'elle entendait par «banalité du mal » — une thèse qu'il qualifiait de slogan —, il écrivait que cette conception du mal entrait en contradiction avec la thèse qu'elle avait développée dans Les origines $d u$ totalitarisme. Arendt lui répond, le 24 juillet 1963, qu'elle a effectivement changé d'idée : elle avance dorénavant que le mal n'est pas radical, mais banal:

À l'heure actuelle, mon avis est que le mal n'est jamais «radical », qu'il est seulement extrême, et qu'il ne possède ni profondeur ni dimension démoniaque. Il peut tout envahir et ravager le monde entier précisément parce qu'il se propage comme un champignon. Il «défie la pensée », comme je l'ai dit, parce que la pensée essaie d'atteindre à la profondeur, de toucher aux racines, et du moment qu'elle s'occupe du mal, elle est frustrée parce qu'elle ne trouve rien. C'est là sa «banalité ». Seul le bien a de la profondeur et peut être radical. (Les origines du totalitarisme 1358)

\section{Eichmann ou la banalité du mal}

Pourquoi Arendt a-t-elle changé d'idée? Nous abondons dans le sens de Bernstein lorsqu'il écrit : «Against Scholem, who states that radical evil and the banality of evil are contradictory, I want to argue for the compatibility of these conceptions of evil» (Hannah Arendt 147). Bernstein argumente en faveur d'un changement de perspectives qui aurait conduit Arendt à modifier sa terminologie. Le fait que son intérêt soit passé de la superfluité à la vacuité de la pensée, aurait conduit Arendt du mal radical à la banalité du mal et surtout à un nouveau cheminement de pensée. Bernstein exprime sa thèse ainsi :

It is as if Arendt had initially felt the need to understand what was unprecedented in the evil that erupted with the advent of twentieth-century totalitarianism. Her response was that never before had there been such a thorough, systematic attempt to change human nature, to make human beings in their plurality, spontaneity, and individuality superfluous. But after the Eichmann trial she became preoccupied with a new and different question: how to account for the "monstrous deeds " committed by persons who in other circumstances seemed so "normal " and "ordinary». (Hannah Arendt 152) 
Nous croyons que Bernstein a tout à fait raison de pointer vers un changement de centre d'intérêt. Nous étayerons son analyse à la lumière de la critique qu'Arendt fait de $\mathrm{Kant}^{10}$.

Il faut mentionner que Henry E. Allison, dans son article «Reflections on the Banality of (Radical) Evil », argumente aussi en faveur d'une continuité entre le mal radical et la banalité du mal. Cependant, sa thèse consiste à dire que la «banalité du mal » pourrait être lue dans une perspective kantienne. Quoique sa brillante analyse mentionne des éléments importants sur les thèses kantiennes, il nous semble qu'Allison n'a pas suffisamment pris en compte les commentaires d'Arendt sur la Critique de la raison pratique. Dana Villa remarque justement qu'une interprétation kantienne d'Arendt peut sembler plausible à première vue, mais qu'elle est en fait insoutenable. Comme il l'explique,

[d] espite her great admiration for Kant, Arendt peremptorily dismisses the suggestion that his practical philosophy can add anything to the discussion of action, let alone clarify the nature of political freedom. [...] This radical disjunction flows, in part, from Arendt's firmly held conviction that the will is an antipolitical faculty. It is not political because it excludes plurality, and plurality is the condition sine qua non of political action and freedom. Hence, "freedom as related to politics is not a phenomenon of the will ». The species of moral causality Kant proposes is prepolitical. (61-62)

Voilà les éléments que nous devons prendre en considération afin de comprendre pourquoi Arendt abandonne la notion de «mal radical ».

Nous avançons qu'Arendt ne renonce pas à son analyse du totalitarisme comme une tentative de rendre les hommes superflus et désolés — ce qu'elle désignait comme le «mal radical ». Ce qu'elle semble plutôt vouloir abandonner, c'est la référence kantienne attachée à l'expression de «mal radical». Nous pensons qu'Arendt a cherché à raffiner sa terminologie afin de mieux rendre compte du phénomène totalitaire. Avant d'aborder directement la distance que prend Arendt face à la philosophie pratique kantienne, il convient de se pencher sur son analyse d'Eichmann. Loin d'aborder le compte rendu du procès d'Eichmann en détails, nous nous 
bornerons à en dégager les éléments essentiels à la compréhension de la notion de «banalité du mal $»^{11}$.

Premièrement, Eichmann représente pour Arendt l'idéal-type de l'homme totalitaire et exemplifie, en ce sens, sa thèse sur la désolation. En effet, l'individu qui n'a plus de rapports authentiques avec les autres travaillera très fort pour devenir un employé consciencieux et acquérir une certaine reconnaissance. Il sera prêt à effectuer n'importe quelle tâche, même l'extermination de millions de personnes, en autant que cette tâche ait l'apparence d'un travail routinier et soit soigneusement organisée. Comme l'écrit Elisabeth Young-Bruehl, « $[n]$ o deeprooted or radical evil was necessary to make the trains to Auschwitz run on time » (Why Arendt Matters 108). Eichmann n'était donc pas possédé du démon, il était tout simplement et banalement un bon père de famille, un employé soucieux de bien faire son travail, ce qui lui valut aussi le titre de «spécialiste ${ }^{12} »$ de la solution finale. Comme l'écrit Arendt dans Eichmann: «Mis à part l'extraordinaire intérêt qu'il manifestait pour son avancement, Eichmann n'avait aucun mobile; et le seul carriérisme n'est pas un crime » (460). Cette absence de mobile constitue un aspect central de la thèse sur la banalité du mal et représente une grande difficulté pour les juges. Comme l'explique Arendt,

[c]et échec des juges de Jérusalem était lié à un autre : leur incapacité à comprendre le criminel qu'ils étaient venus juger. [...] Il eût été réconfortant de croire que Eichmann était un monstre [...]. L'ennui, avec Eichmann, c'est précisément qu'il y en avait beaucoup qui lui ressemblaient et qui n'étaient ni pervers ni sadiques, qui étaient, et sont encore, effroyablement normaux. Du point de vue de nos institutions et de notre éthique, cette normalité est beaucoup plus terrifiante que toutes les atrocités réunies, car elle suppose (les accusés et leurs avocats le répétèrent, à Nuremberg, mille fois) que ce nouveau type de criminel, tout hostis humani generis qu'il soit, commet des crimes dans des circonstances telles qu'il lui est impossible de savoir ou de sentir qu'il a fait le mal. (443-444) 
Eichmann n'avait aucun motif ou aucune raison personnelle pour détester les Juifs. Il n'avait, selon Arendt, aucune conviction idéologique, il n'avait pas sa carte du parti nazi et ne connaissait pas très bien leur programme. Il ne prenait jamais de décisions de façon autonome et s'arrangeait toujours pour agir en fonction d'ordres reçus. Lors du procès, il répondit aux questions par des phrases toutes faites, des clichés. En fait, il a fit siennes les règles de langage (Sprachregelungen) édictées par le régime et il fut même fier de dire que le langage administratif était le seul qu'il connût. Dans La pensée, Arendt écrit que ces clichés « ont pour fonction reconnue, socialement, de protéger de la réalité » (19).

Eichmann est dépeint par Arendt comme l'archétype de l'homme superflu, c'est-à-dire un homme sans croyance, sans profondeur et facilement remplaçable par quiconque eût accepté de faire sa tâche. La thèse qu'elle propose est qu'Eichmann fait preuve d'une vacuité de la pensée. Il n'est pas corrompu par nature, il souffre d'un manque de rapport au monde. La mal qu'il a imposé aux autres est sans motif et surtout sans profondeur ontologique. Ce mal est donc banal, c'est-à-dire accompli sans jugement. Eichmann est tout simplement incapable de se mettre à la place des autres, de faire preuve de pensée élargie au sens kantien du terme. Dans l'extrait cité précédemment, Arendt remarque que cette normalité est encore plus terrifiante que la perversion ou le démoniaque. Ce «nouveau type de criminel » défie la compréhension traditionnelle de la criminalité. Un autre extrait d'Eichmann met en relief la difficulté majeure de notre compréhension du crime :

Tous les systèmes juridiques modernes supposent que pour commettre un crime il faut avoir l'intention de faire le mal. Les peuples civilisés s'enorgueillissent tout particulièrement de ce que leur jurisprudence prend en considération ce facteur subjectif. Quand cette intention est absente, quand, pour une raison ou une autre, fût-ce l'aliénation morale, la faculté de distinguer le bien du mal est atteinte, nous pensons qu'il n'y a pas eu de crime. (445) 
En assistant au procès Eichmann, Arendt confirme certaines intuitions que Karl Jaspers avait eues en 1946. Commentant le dernier livre de Jaspers, Schuldfrage, Arendt disait que les actes des nazis ne pouvaient pas être compris comme des crimes. Dans la réponse qu'il adressa à Arendt, le 23 octobre 1946, Jaspers écrivit :

votre conception m'inquiète un peu du fait que la faute qui dépasse toute faute criminelle acquiert inévitablement une certaine « grandeur » - une grandeur satanique, qui, pour ce qui est des nazis, est aussi loin de moi que le discours sur le «démonisme » de Hitler et autres choses de cette sorte. À mon avis, c'est parce qu'il en a vraiment été ainsi qu'il faut voir les choses dans toute leur banalité, dans leur prosaïque nullité - les bactéries peuvent provoquer des épidémies anéantissant des populations entières et ne resteront pourtant que des bactéries. (Arendt-Jaspers, Correspondance 110-111)

À quoi Arendt répondit qu'elle s'approchait «dangereusement d'une "grandeur satanique" » (Correspondance 120) qu'elle voulait précisément éviter. L'aspect important, qu'elle n'arrivait pas encore à formuler à cette époque, était qu'il devait exister une différence entre ceux qui commettent intentionnellement des crimes et les nazis qui avaient commis des actes dépassant toute utilité. Comme elle le soulignait alors, «les déportations ont été très nuisibles aux opérations de guerre » (Correspondance 120). L'extrait de la lettre de Jaspers que nous venons de citer rappelle étrangement la réponse que donnait Arendt à la critique de Scholem, quoique Arendt n'ait jamais reconnu l'influence de son maître dans la formulation de sa notion de banalité du mal. Quoi qu'il en soit, une chose apparaît clairement : Arendt était déjà consciente, en 1946, qu'il fallait éviter toute «grandeur satanique» et toutes tentatives de mythifier l'horreur. D'autant plus qu'elle comprenait que «[d]ans le Troisième Reich, le mal avait perdu cet attribut par lequel on le reconnaît généralement : celui de la tentation » (Eichmann 244).

Le passage du mal radical à la banalité du mal doit ainsi être compris comme une tentative visant à éviter que les horreurs totalitaires ne prennent la forme d'une grandeur satanique. Le ton ironique qu'elle donna à son compte rendu du procès — et qui fit l'objet de 
vives critiques, notamment de la part de Scholem — était peut-être calculé. L'ironie permet en effet de créer une mise à distance plutôt qu'une identification. Depuis les écrits du marquis de Sade, nous savons que le péché et la perversion peuvent provoquer une certaine fascination. L'attrait de l'interdit, du reste, est connu depuis le récit biblique de la Chute. Arendt cherchait ainsi à éliminer toute possibilité d'identification et d'attrait au meurtre des Juifs, à la superfluité des hommes, voire à l'éradication de l'humanité des hommes. Susan Neiman remarque très justement à propos de l'ironie :

It's a tone that creates distance in place of desire. Like Brecht, Arendt argued that the comedy undermines evil more effectively than does tragedy. The diabolic can be ambiguous; the ridiculous is not. To call evil banal is to call it boring. And if it is boring, its appeal will be limited. A fungus, after all, is rarely erotic. (302)

Arendt résumait ainsi sa position sur Eichmann dans le post-scriptum au compte rendu du procès :

Eichmann n'était pas stupide. C'est la pure absence de pensée - ce qui n'est pas du tout la même chose - qui lui a permis de devenir un des plus grands criminels de son époque. Cela est «banal» et même comique : avec la meilleure volonté du monde on ne parvient pas à découvrir en Eichmann la moindre profondeur diabolique ou démoniaque. (460)

Arendt découvrait ainsi que les sources du mal ne sont pas mystérieuses, profondes ou diaboliques : elles sont plutôt à la portée de tous les hommes. Il n'est pas nécessaire d'invoquer des forces surnaturelles afin de comprendre le mal totalitaire. Si le mal n'a pas de profondeur ontologique et s'il est banal, il est donc en notre pouvoir de le combattre en exerçant notre faculté de penser et de juger. Voilà le pari que se donne Arendt lorsque, à la fin de sa vie, elle se penche sur la vie de l'esprit. 


\section{Réflexions critiques sur la philosophie morale kantienne}

Arendt commence son ouvrage sur la vie de l'esprit en rappelant que c'est le procès Eichmann qui l'a poussée à s'intéresser à la question des activités de l'esprit. Elle sentait bien que sa notion de «banalité du mal » prenait à rebours la pensée traditionnelle. Comme l'exprime si bien Young-Bruehl, dans La vie de l'esprit, Arendt «looked back on the entire Western philosophical legacy of investigation into how we think, will, and judge and rejected almost all of it» (Why Arendt Matters 159-160). La notion de «banalité du mal » la plaçait devant une question qui semblait inconnue de la tradition littéraire, théologique et philosophique, à savoir : «Le mal (par omission aussi bien que par action) est-il possible quand manquent non seulement les "motifs répréhensibles" (selon la terminologie légale) mais encore les motifs tout court, le moindre mouvement d'intérêt ou de volonté? »(La pensée 19). Bien que de nombreuses autres questions motivent l'investigation sur les facultés de l'esprit, nous nous intéresserons à celle-ci, puisqu'elle nous permettra de saisir la raison pour laquelle Arendt abandonne la notion de «mal radical ».

Jusqu'à présent, nous avons vu que le mal totalitaire ne peut être compris ni en termes d'intention ou de motif, ni en termes de nature pervertie et cela, même lorsqu'il est question de «mal radical ». Cette première constatation marque déjà la distance que prend Arendt face à Kant et permet de constater qu'elle utilise l'expression kantienne de « mal radical » en la vidant de son sens.

Selon Kant, la notion de «mal radical » est liée à une perversion de la volonté. Il s'agit en fait de la propension humaine à choisir comme détermination de la volonté des maximes autres que celle de la loi morale. L'adjectif « radical » marque le fait que cette propension est enracinée

dans la nature humaine, qu'elle a donc une certaine profondeur ${ }^{13}$. L'échec de la volonté à adopter 
une maxime morale a une « racine transcendantale », mais l'homme, par son libre-arbitre, a tout de même la possibilité de choisir entre une bonne ou une mauvaise maxime. Du moment où la volonté choisit une maxime autre que celle de la loi morale, elle est dans le mal ${ }^{14}$. Le mal moral n'est donc pas lié aux conséquences mauvaises d'une action, mais à l'intention de l'agent ou, pour le dire plus exactement, au principe de détermination de la volonté. Ainsi, même une action qui aurait de bonnes conséquences et qui respecterait la légalité serait moralement mauvaise si son principe de détermination était autre que celui de la loi morale. Kant insiste à plusieurs reprises sur cet aspect qui fonde aussi la différence entre la lettre et l'esprit de la loi, c'est-à-dire entre la légalité et la moralité. Relisons un extrait de la Critique de la raison pratique :

Un autre principe subjectif ne doit (muss) pas être pris pour mobile, car autrement l'action peut sans doute se présenter, comme le prescrit la loi, mais bien que conforme au devoir, elle n'a pas lieu par devoir, l'intention, dont il s'agit essentiellement pourtant dans cette législation, n'en est pas morale. (86)

Kant est catégorique : la moralité est essentiellement liée à l'intention. Comme le précise Alexis Philonenko, «la volonté (der Wille) qui ne s'applique à rien d'autre qu'à la loi ne peut être dite libre ou non-libre parce qu'elle ne porte pas immédiatement sur les actions, mais sur la législation pour les maximes des actions (par conséquent la raison pratique elle-même) » (156). C'est pour cette raison que la philosophie pratique de Kant peut être qualifiée de prépolitique, comme le soulignait Villa, et c'est aussi pour cette raison qu'elle n'intéresse pas Arendt.

Il faut ici rappeler qu'Arendt cherchait, en quelque sorte, à justifier son choix d'utiliser la troisième Critique plutôt que la deuxième, afin de parler de la philosophie politique de Kant. Elle savait combien sa proposition pouvait sembler controversée. Selon elle, ce qui faisait le plus défaut à la deuxième Critique et, inversement, ce qui faisait la force de la troisième, c'était la question de la pluralité. Dans la Critique de la raison pratique, l'être rationnel est le seul juge de 
la moralité, c'est-à-dire que seul l'examen de conscience permet de révéler si l'esprit de la loi a été respecté. À la suite du procès d'Eichmann, Arendt comprend que la voix de la conscience est en fait une «actualization of the consciousness in the activity of thinking 》 (Kohn, Evil 8). Si la personne souffre d'une vacuité de la pensée, elle ne pourra plus actualiser ce processus réflexif et sa conscience sera déficiente (Eichmann 239). Mais la Critique de la faculté de juger implique nécessairement une pluralité de spectateurs et se fonde sur le principe de publicité. Dans son cours Kant's Political Philosophy, donné à l'Université de Chicago en 1964 et en 1970, Arendt disait justement que la philosophie pratique kantienne porte sur l'intention. Elle posait alors une distinction entre l'intention et la signification - une distinction qui recoupe celle entre le début et la fin d'une action. Étant donné qu'Arendt se questionnait sur la politique, elle s'intéressait à ce qui apparait dans le monde, la fin de l'action et sa signification. Comme elle l'écrivait pour son cours sur la philosophie politique kantienne,

Kant in his moral philosophy starts with the beginning, only the will can be good, the intentions. But these intentions never appear. Hence: when he has to judge the world of appearances he has to find a different set of concepts and these concepts are in contradiction to his moral philosophy. (032252)

Dans le même cours, elle ajoutait que dans le domaine de la moralité, «[w]e don't consider the consequences, they are immaterial for the validity of the Law, and we are concerned only with not contradicting ourselves» $(032271)^{15}$. Arendt pose ainsi une distinction tranchée entre la moralité et la politique, et donc entre le mal moral et le mal politique. Elle ne s'intéresse qu'à ce qui apparaît dans le monde : les actions et leurs conséquences. D'autre part, les critères pour juger de l'apparaître ne seront pas les mêmes que pour juger de la moralité des intentions, les premiers devant nécessairement être communicables. Comme elle l'exprime, «en politique, pour autant qu'elle se distingue de la morale, tout dépend de la "conduite publique" » (Juger 37). 
C'est en ce sens qu'il faut comprendre ses propos dans Eichmann: « Nous ne nous intéressons qu'à vos actes. Votre vie intérieure, qui n'était peut-être pas celle d'un criminel, et les potentialités criminelles de ceux qui vous entouraient, nous importent peu » (447). Est-il besoin de rappeler qu'Arendt ne s'est jamais réellement intéressée à la question de la vie intérieure, si ce n'est que pour déplorer le fait que l'idée de liberté intérieure ait obscurci le sens de la liberté politique? En définitive, nous pouvons dire que peu importe les intentions, ce sont les actes qui comptent. Et pour juger les actes, la raison pratique n'est d'aucune aide : «Le jugement n'est pas la raison pratique; la raison pratique "raisonne" et me prescrit ce que je dois et ne dois pas faire; elle instaure la loi et est identique à la volonté. La volonté énonce des commandements et parle à l'impératif»(Juger 32). Le choix d'Arendt est clair : ce n'est pas la raison pratique kantienne qui pourra aider à juger le mal politique, mais bien une réappropriation de la faculté de juger kantienne.

\section{La vie de l'esprit ou l'amor mundi}

Afin d'approfondir la conception arendtienne du mal, il s'avère utile de considérer les sources augustiniennes de sa pensée ${ }^{16}$. Tout comme elle le fit avec Kant, Arendt entre en dialogue avec saint Augustin, l'approuvant et le désapprouvant tout à la fois. Nous nous attarderons sur deux notions augustiniennes : la compréhension du mal comme privatio boni et la résolution du conflit de la volonté par l'amour. Ces deux notions nous permettront d'étayer notre thèse sur l'articulation entre le «mal radical » et la «banalité du mal ».

Premièrement, le mal est décrit chez Arendt en termes essentiellement négatifs, c'est-àdire selon ce qui est détruit et ce qui fait défaut. En effet, nous avons vu que le «mal radical » est lié à la destruction du monde commun, à la superfluité des hommes et à l'éradication de leur 
humanité. La «banalité du mal » se rapporte quant à elle à la vacuité de la pensée et à l'impossibilité de se mettre à la place des autres. Ainsi, Charles T. Mathewes souligne très justement que la conception arendtienne du mal, malgré son caractère moderne, a une résonance augustinienne. Comme il l'écrit, cette conception «sees evil as the negation of genuine human goods, and as the contradiction of the human agent to something ultimately sub-human » (169). Par ailleurs, il ajoute que pour Arendt, «only amor mundi can provide us with the genuine goods of human existence » (179). Voilà les deux éléments importants à prendre en considération : le mal comme négation et l'importance de l'amor mundi. Évidemment, toute la difficulté provient du fait qu'Arendt ne se réfère pas directement à saint Augustin dans Eichmann. Le lien établi par Mathewes n'en demeure pas moins pertinent: Arendt reprendrait l'idée augustinienne du mal comme privatio boni, ou dans des termes plus arendtiens, le mal serait lié à un manque d'amor mundi.

Poursuivons avec un deuxième élément qui permet d'aborder le rapport entre la volonté et le mal, à savoir le commentaire de Mathewes sur le caractère d'Eichmann. Il écrit: «Eichmann's crimes were not rooted in a wicked character : he has no character to be wicked» (167). Ce commentaire évoque celui que fit Arendt sur Martin Heidegger. Dans Why Arendt Matters, Young-Bruehl rappelle qu'à la toute fin de sa vie, Arendt se risqua à une réflexion critique sur la pensée de Heidegger. Cette réflexion est cependant entachée par une certaine déception quant au fait que Heidegger ait délaissé le terrain de l'action. Arendt fut formée par Heidegger et, à bien des égards, elle est héritière de sa critique de la modernité. Elle approuve sa critique de la subjectivité et comprend les difficultés et les dangers de la volonté ${ }^{17}$. Cependant, elle n'endosse pas sa répudiation de la volonté et sa fuite dans la pensée. Young-Bruehl résume magistralement le cœur de la question : 
Such a conception could be seen as Heidegger's repudiation of his own disastrous entry into the political realm, his membership in the Nazi Party, but it carried the further implication that no one could enter political life without giving up the only kind of uncorrupted life there is, the life of the thinker. There was certainly no notion here that the will can constructively shape character, training a person to make choices, to act. It was this view of will and action that had led Arendt to diagnose him in a letter to Jaspers soon after the war as lacking character, "in the sense that he literally has none and certainly not a particular bad one ». [...] He failed to see that willing is at peace and non dominating when it is loving. (195-196)

C'est précisément dans la pensée de saint Augustin qu'Arendt a trouvé ce modèle de volonté, une volonté qui n'est pas souveraine et qui ne plonge pas l'esprit dans le conflit. Dans Le vouloir, Arendt s'attarde longuement sur saint Augustin puisque selon elle, il est le «premier philosophe de la Volonté ». Elle découvre avec saint Augustin que la volonté est libre : elle n'est pas déterminée par les objets extérieurs, elle est à elle-même sa propre cause. Selon saint Augustin, ce qui cause le conflit de la volonté n'est pas le conflit entre la chair et l'esprit, comme c'était le cas avec saint Paul. Le conflit dans la volonté émerge d'une fissure à l'intérieur même de la volonté. Il tient à la nature de la volonté et surtout de son mode d'expression, à savoir la forme impérative. Comme l'explique Arendt, «[d]ans tout acte de volonté se trouvent impliqués un je-veux et un je non-veux. Ce sont les deux volontés dont saint Augustin dit que leurs “conflits" lui "ravageaient l'âme" »(109). C'est sur cette compréhension augustinienne du conflit de la volonté qu'Arendt s'appuie afin de rejeter toute idée de souveraineté basée sur la volonté, tant au niveau politique qu'au niveau de la vie de l'esprit. Le commandement provoque toujours sa contrepartie dans la résistance, plongeant le moi-voulant dans un enfermement sur lui-même, précisément causé par sa nature réflexive.

Le conflit de la volonté n'est pas résolu par la grâce chez saint Augustin, mais par l'amour. Dans les Confessions, «il identifie dans l'Amour la volonté unifiante dernière qui détermine la conduite d'un homme» (Vouloir 115). Arendt poursuit en disant que «[1]a 
pesanteur de l'âme, l'essence de qui est quelqu'un, inscrutable à l'œil humain, devient manifeste dans cet amour»(Vouloir 116). Dans De la Trinité, saint Augustin explique que prise en ellemême, la volonté est source de conflit, mais que prise dans son interrelation avec les autres facultés de l'esprit, elle peut devenir source d'unité. Arendt commentait dans son cours The History of the Will, donné à la New School en 1971 :

That means that the puzzling fact of the will, that must will to will and, because of the imperative nature of the will, produces its own counterwill, is no longer the decisive quality of the will. This is also the reason why the will in this context has lost its voice of command. The will unites, it no longer commands. And because it unites, it can be identified with love. (024699)

L'amour devient ainsi le principe de la volonté, la rédemption de la volonté et une force plus permanente que les différentes volitions : la volonté-amour «provoque une permanence dont l'esprit semble autrement incapable » (Vouloir 125). L'Amour est conçu comme une sorte de volonté durable qu'Arendt associe au «moi qui dure » de John Stuart Mill, une permanence malgré les fluctuations entre le vouloir et le non-vouloir. La volonté-amour permet une harmonie dans l'esprit, puisque ainsi transformée, la volonté est exempte de conflits. Il faut aussi noter que chez saint Augustin, l'individualité s'exprime par la volonté: elle est un principium individuationis. Comme le résume Arendt dans Le vouloir,

[1]'Amour, vu par saint Augustin, exerce son influence par le «poids » — «quand à la volonté, elle joue le rôle de poids » - elle amplifie l'âme et jugule ainsi ses fluctuations. L'homme ne devient pas juste en sachant ce qui est juste, mais en aimant la justice. (125)

Arendt a évidemment des critiques à formuler à l'endroit de la conception augustinienne de la volonté, notamment quant au fait qu'il n'a pas tiré toutes les conséquences de ses spéculations. Augustin aurait dû voir que la liberté de la volonté n'est pas seulement le liberum arbitrium, libre-choix entre vouloir et non-vouloir, mais la liberté entendue comme «pouvoir capable de commencer par lui-même»(Vouloir 131), retournant ainsi à Kant. Malgré ces 
critiques, Arendt a compris avec saint Augustin que la volonté transformée en amour forme le caractère et instruit le moi quant aux choix à faire entre différents projets. La volonté n'est pas directement le moteur de l'action, puisque l'activité de volition doit cesser pour que l'on puisse agir : l'action met fin au conflit de la volonté. Cependant, l'action se fait sur la base de notre caractère, et si le moi est formé par l'amour, les actions risquent d'être plus justes. Comme Arendt l'exprimait dans la conclusion de La pensée, préparant ainsi le terrain pour Le vouloir,

la volition est l'aptitude intérieure grâce à laquelle les hommes décident «qui » ils vont être, sous quelle forme ils veulent se montrer dans le monde des phénomènes. Autrement dit, c'est la volonté, axée sur les projets et non les objets, qui, en un sens, crée la personne qu'on pourra louer ou blâmer, et en tout cas tenir pour responsable, non seulement de ses actes, mais aussi de son « Être » tout entier, de son caractère. (240)

Ce que nous pouvons appeler «l'événement Eichmann » a plongé Arendt dans une série d'interrogations sur les facultés de l'esprit. Dans La vie de l'esprit, elle ne fait pas de lien direct entre Eichmann et la volonté. Cependant, il nous semble plausible que si la répudiation de la volonté n'est pas une solution pour Arendt, comme elle le fut pour Heidegger, c'est parce qu'Arendt a vu Eichmann abdiquer sa volonté personnelle pour adopter la volonté de son Führer. En fait, Eichmann avait repris l'impératif catégorique kantien, mais en pratique, il l'avait déformé. Il identifiait sa volonté personnelle avec la source de la loi — dans son cas, la volonté du Führer (Eichmann 223-223). En renonçant à sa volonté personnelle, Eichmann se croyait audessus de toute responsabilité. La volonté totalitaire, liée au commandement, n'avait pas non plus le caractère de la volonté unifiée par l'amour. Dans le cas d'Eichmann, cette répudiation de la volonté n'a pas été au grand bonheur de la pensée, puisque Arendt remarque qu'il souffrait d'une absence de pensée.

Nous croyons que la notion de caractère formée par la volonté-amour aurait joué un rôle important dans la pratique du jugement. Malheureusement, Arendt est décédée avant d'avoir pu 
terminer sa Vie de l'esprit. Peut-être en serait-elle venue à une articulation des différentes facultés de l'esprit, comme elle avait su le faire, dans Condition de l'homme moderne, avec le travail, l'œuvre et l'action. L'amor mundi, le souci du monde qui préside à la conservation du monde commun et de la pluralité, aurait peut-être inspiré l'harmonie et le bon fonctionnement de la vie de l'esprit. Une chose est certaine, Eichmann manquait d'amor mundi ${ }^{18}$, de cet amour qui fait que nous partageons le monde avec les autres et que nous tissons des liens entre nous — et c'est sur ce principe même qu'Arendt le condamne. Elle conclut ainsi Eichmann :

Et parce que vous avez soutenu et exécuté une politique qui consistait à refuser de partager la terre avec le peuple juif et les peuples d'un certain nombre d'autres nations comme si vous et vos supérieurs aviez le droit de décider qui doit et ne doit pas habiter cette planète - nous estimons que personne, qu'aucun être humain, ne peut avoir envie de partager cette planète avec vous. C'est pour cette raison, et pour cette raison seule, que vous devez être pendu. (448)

\section{Conclusion}

En définitive, nous défendons l'idée d'une continuité dans l'œuvre d'Arendt. Hannah Arendt ne se dément pas lorsqu'elle abandonne l'expression de «mal radical » pour adopter celle de «banalité du mal». Afin de rendre justice à l'entreprise arendtienne de compréhension du phénomène du mal totalitaire, il convient même de réunir le «mal radical » et la «banalité du mal ». Nous pouvons ainsi concilier les deux expressions en disant que le mal est radical pour le monde commun parce qu'il vise totalement sa destruction, mais ce mal est accompli de manière banale par l'individu, c'est-à-dire sans la profondeur des racines intentionnelles. Cette formulation paradoxale éclaire le phénomène du mal politique, à savoir l'idée d'un mal qui n'a pas de caractère satanique ni de tentation et qui a toutefois des conséquences sur la totalité de la vie. Cependant, afin de réconcilier ces deux concepts, il importe de vider l'expression de «mal 
radical » de sa référence kantienne. La radicalité ne vient plus désigner la propension humaine à choisir une autre maxime que celle de la loi morale, mais le caractère total d'un mal qui s'attaque à la nature humaine elle-même en tentant d'abolir les racines du vivre-ensemble, c'est-à-dire la spontanéité et la liberté humaine. Cette compréhension révèle la responsabilité des hommes dans ce mal et, du même coup, l'espoir qu'il est en notre pouvoir de le combattre. Contrairement à ce que ses détracteurs ont cru, Arendt n'a jamais pensé qu'Eichmann n'était pas responsable de ses actes. Comme le souligne si justement Mathewes,

$[w]$ e cannot despair, because there is nothing to despair about; our opponents are not demonic agents of an antihuman conspiracy, but rather the banal bureaucrats of some potentially subhuman world empire. This account of evil gives us hope, for it suggests that our only opponents are ourselves made less real, «banal ». (170)

Il s'avère important de maintenir la tension entre le «mal radical » et la «banalité du mal », deux concepts s'imbriquant l'un dans l'autre. Cette tension représente aussi la dialectique de l'homme dans le monde, un être dont la vie de l'esprit est affectée par son rapport au monde. En effet, la disparition du monde commun affecte la faculté de penser, de vouloir et de juger des hommes. Et sans le partage des idées dans le monde commun, les hommes risquent d'aggraver la destruction du tissu des relations humaines. Nous avons la responsabilité de ce monde, dont il nous faut prendre soin. C'est pourquoi il faut garder la mémoire du mal radical dans sa perspective de destruction de la pluralité humaine et dans sa tentative de rendre les hommes superflus. Ce devoir de mémoire doit nous permettre d'empêcher les futurs génocides qui pourraient être accomplis comme de banales procédures administratives par des gens qui ne se soucient que de leur avancement personnel — des gens à qui manque l'amor mundi. 


\section{Notes}

${ }^{1}$ Il existe plusieurs excellents commentaires sur le rôle de la pensée et du jugement dans la question du mal. Nous renvoyons à «Essais interprétatifs » par Ronald Beiner et Myriam Revault d'Allones, Juger (1991); Dana Villa, Arendt and Heidegger (1996); Françoise Collin, L'homme est-il devenu superflu? (1999); Sylvie Courtine-Denamy, Le souci du monde (1999); Étienne Tassin, Le trésor perdu (1999); Ronald Beiner et Jennifer Nedelsky (dir.), Judgment, Imagination, and Politics (2001); Miguel Abensour, Hannah Arendt contre la philosophie politique? (2006); Jerome Kohn, « Evil : The Crime against Humanity ».

2 Il est à noter qu'Arendt eut beaucoup de difficulté à trouver un éditeur français pour son ouvrage qui fut finalement démantelé par les éditeurs français. La dernière partie, «Le système totalitaire », parut en 1972 aux éditions du Seuil, suivie de «Sur l'antisémitisme », chez Calmann-Lévy en 1973, et enfin, «L'impérialisme» parut chez Fayard en 1982. En 2002, l'éditeur français Gallimard réunit finalement les trois parties de l'ouvrage sous le titre Les origines $d u$ totalitarisme en y ajoutant aussi Eichmann à Jérusalem et plusieurs textes complémentaires. À ce sujet, les commentaires de Pierre Bouretz sont très pertinents, particulièrement quant à la réception de l'œuvre en France.

${ }^{3}$ Pour une brillante analyse des processus de la modernité à l'œuvre dans les schèmes du totalitarisme, voir l'ouvrage d'Étienne Tassin, Le trésor perdu. Hannah Arendt l'intelligence de l'action politique, particulièrement le chapitre III de la deuxième partie.

${ }^{4}$ Nous référons au chapitre III de la troisième partie de l'ouvrage pionnier d'André Enegrén, $L a$ pensée politique de Hannah Arendt, pour une mise en lumière de cette marche vers la désolation reliant les trois parties des Origines du totalitarisme.

${ }^{5}$ Nous avons tenu à citer l'extrait de cette lettre en anglais, puisque la traduction française omet le terme «motives » qui nous paraît capital pour comprendre le passage de l'expression «mal radical » à celle de «banalité du mal ». La traduction de cette lettre peut être consultée dans la correspondance entre Hannah Arendt et Karl Jaspers (1926-1969), 243.

${ }^{6}$ Dans Le trésor perdu, Étienne Tassin explique qu'Arendt ne croit pas que la nature humaine puisse être réellement changée. Elle souligne seulement que la tentative de changer la nature humaine conduit inévitablement à sa destruction. Tassin écrit : "La "nature" humaine n'a de sens qu'au titre de ses conditions existentiales et de son existence historique et non comme essence éternelle: il n'y a pas plus, pourrait-on dire, d'essence de l'homme hors de ses conditions historiques d'existence qu'il n'y a d'essence du totalitarisme hors de son effectuation historique, c'est-à-dire hors d'un processus de cristallisations d'éléments, en eux-mêmes non totalitaires » (141). C'est précisément la condition de pluralité qui était visée par le régime totalitaire, et surtout la réponse humaine à cette condition, la spontanéité et l'action. Dans Condition de l'homme moderne, Arendt changera justement sa position en substituant la notion de « condition humaine » à celle de «nature humaine »(Condition de l'homme moderne 44-46). 
7 Dans Le trésor perdu, Tassin explique que la désolation «est l'épreuve conjointe du déracinement et de la superfluité » (177). Il lie de manière magistrale la privation d'un sol (déracinement) à la privation du soi, et interprète ainsi la privation d'un sol comme une perte de lien humain. Sans ce lien humain, l'individu perd la capacité de devenir une singularité: «Il n’y a de singularité que depuis l'interpellation plurielle qui me fait "irremplaçable" » (179). Pour une exploration du thème de la désolation, voir Le trésor perdu, particulièrement les pages 176-185.

${ }^{8}$ Dans La pensée, Arendt explique que toutes les activités mentales nécessitent une forme de retrait des phénomènes, particulièrement de ce que nous recevons du monde par les sens. Cependant, elle spécifie que « seule la pensée, de par sa tendance à généraliser, c'est-à-dire son goût spécial du général en tant qu'il diffère du particulier, est portée à se mettre totalement à l'écart du monde » (92). Cet aspect constitue aussi un argument en faveur de la distinction entre la pensée et le jugement. La pensée, en raison de sa tendance à se retirer totalement du monde et de son goût prononcé pour le général plutôt que le particulier, ne peut pas être une faculté politique. Le retrait du monde du jugement est celui du spectateur qui observe les événements sans y être impliqué et a donc le recul nécessaire à l'impartialité. Il est possible que l'aspect qu'Arendt voulait mettre en relief en parlant de la pensée comme d'une condition préparant le jugement était, plus précisément, la capacité de représentation et la faculté d'imagination (voir La pensée 85-97).

${ }^{9}$ Cet échange de lettres entre Gershom Scholem et Hannah Arendt a été traduit en français et reproduit dans l'édition Gallimard des Origines du totalitarisme, aux pages 1342 à 1348 (pour la lettre de Scholem) et 1353 à 1358 (pour la réponse d'Arendt).

${ }^{10}$ Dans Hannah Arendt and the Jewish Question, Bernstein écrit : «This passage shows how strikingly Arendt departs from the traditional, entrenched conception of evil understood as a manifestation of "evil motives" » (151). Bernstein ne fait que constater cette distance que prend Arendt face à la tradition, sans élaborer. Il est donc important de développer cette idée en montrant qu'en attaquant la question de l'intentionnalité, c'est à la deuxième critique kantienne que s'attaque Arendt.

11 La parution d'Eichmann à Jérusalem provoqua une virulente controverse. En fait foi l'abondance de documents du « Adolf Eichmann File, 1938-1968 » des «Hannah Arendt Papers at the Library of Congress ». Étant donné son ampleur, nous ne pourrons malheureusement pas reprendre les enjeux de cette controverse. Du reste, de nombreux commentateurs l'ont déjà fait, dont Bouretz dans son introduction à Eichmann dans l'édition Gallimard des Origines $d u$ totalitarisme (979-1013); Richard J. Bernstein, Hannah Arendt and the Jewish Question (154178); et Elisabeth Young-Bruehl, Hannah Arendt (ch. 8).

${ }^{12}$ Soulignons que l'excellent film de Rony Brauman et Eyal Sivan, Un spécialiste. Portrait d'un criminel moderne (1999), est construit à partir d'images d'archives du procès d'Eichmann à Jérusalem et s'inspire de la réflexion arendtienne sur la banalité du mal. On y voit à l'œuvre l'effroyable banalité du mal. 
${ }^{13}$ Comme le résume Philonenko, «[1]e mal radical, dont Bohatec a sondé les plus profondes racines tant métaphysiques que psychologiques est la simple impuissance à rejoindre la loi. En ceci réside sa racine transcendantale » (L'œuvre de Kant 158).

${ }^{14}$ À ce sujet, voir l'ouvrage de Richard J. Bernstein, Radical Evil. A Philosophical Interrogation.

${ }^{15}$ Dana Villa souligne très justement que Socrate avait proposé le principe de non-contradiction comme critère éthique. La notion socratique est basée sur l'idée d'harmonie dans le «moi », conservant ainsi la pluralité fondamentale entre moi et moi-même nécessaire au dialogue de la pensée. Cependant, dans sa forme kantienne, le principe de non-contradiction perd l'aspect de pluralité. Comme le formule Villa, «[s]o construed, the will in its legislative aspect eliminates all reference to plurality, whether internal or external. What matters is that the will will itself, that it will its own rational (universal) nature » (65).

${ }^{16}$ Nous devons beaucoup pour cette section à Charles T. Mathewes qui, dans son ouvrage Evil and the Augustinian Tradition, invite à considérer les sources augustiniennes de la pensée arendtienne. Il est impossible, dans le contexte de notre réflexion, de discuter en profondeur de la thèse de Mathewes. Cette dernière consiste à déplorer le fait qu'Arendt n'ait repris que partiellement la pensée de saint Augustin. Selon lui, cette reprise partielle présente de graves difficultés pour la pensée arendtienne et «ends up rendering the ontology unsustainable, and the anthropology unintelligible»(196). Mathewes touche ici à l'une des grandes difficultés du penser arendtien. Arendt se sent dans une brèche du temps et elle sait qu'elle doit à la fois penser dans et à la rupture du fil de la tradition - une rupture qui empêche précisément toute reprise intégrale des œuvres du passé. Comme le souligne si bien Pierre Bouretz, « elle veut ici dire au sujet de la philosophie que l'on n'en peut conserver que des "fragments" » (dans Origines du totalitarisme 75$)$.

17 Dans Le vouloir (199-223), Arendt explique que Heidegger en est venu à comprendre la volonté, entendue comme volonté de régir et de dominer, comme une sorte de péché originel. Selon Heidegger, cette volonté de puissance est obsédée par le futur : elle veut en devenir maître. Une telle volonté cherche à forcer l'homme à l'oubli, puisque pour vouloir le futur, elle doit oublier le passé. La volonté veut détruire son passé, puisqu'elle est impuissante face à lui : elle ne peut vouloir en arrière. La volonté devient ainsi destructive par nature. Arendt partage cette critique de la volonté de domination moderne et ses implications technologiques, elle sait que le moi-voulant peut chercher à anéantir la pluralité. Cependant, elle sait aussi qu'il y a une rédemption à la volonté et que la volonté joue un rôle dans la formation du caractère. Elle n'approuve donc pas sa répudiation dans un vouloir ne-pas-vouloir.

18 Elisabeth Young-Bruehl, dans Cherishment (229-231), reprend l'analyse arendtienne de la désolation et de la «banalité du mal» d'Eichmann en termes psychanalytiques. Elle utilise une méthode basée sur le concept japonais amaeru et une approche faisant du « chérir » le fondement émotionnel d'une personne. Son analyse renforce l'idée d'un lien entre l'amour, la formation du caractère et la manière dont la volonté opère. 


\section{Ouvrages cités}

Abensour, Miguel. Hannah Arendt contre la philosophie politique? Paris : Sens \& Tonka, 2006.

Allison, Henry E. «Reflections on the Banality of (Radical) Evil. A Kantian Analysis ». In M. P. Lara (dir.). Rethinking Evil. Contemporary Perspectives. Berkeley (CA)/ London: University of California Press, 2001.

Arendt, Hannah. « The Concentration Camps ». Partisan Review, Vol. 15, n 7, 1948: 743-763.

-. Condition de l'homme moderne. Trad. G. Fradier. Paris : Calmann-Lévy, 1983 (coll. Pocket/Agora).

—. Eichmann à Jérusalem. Rapport sur la banalité du mal. Trad. A. Guérin. Paris : Gallimard, 1997 (coll. Folio histoire).

-. Essays in Understanding. 1930-1954 Formation, Exile, and Totalitarianism. New York: Schocken Books, 1994.

—. L’impérialisme. Trad. M. Leiris. Paris : Fayard, 1983 (coll. Points politique).

—. Juger. Sur la philosophie politique de Kant. Trad. M. Revault d'Allones, Paris : Seuil, 1991 (coll. Libre examen).

—. Les origines du totalitarisme. Eichmann à Jérusalem. Dir. P. Bouretz. Paris : Gallimard, 2002 (coll. Quarto).

—. Responsibility and Judgment. New York : Schocken Books, 2003.

—. Sur l'antisémitisme. Trad. M. Pouteau, Paris : Calmann-Lévy, 1973 (coll. Points essais ).

—. Le système totalitaire. Trad. J.-L. Bourget, R. Davreu et P. Lévy. Paris : Seuil, 1972 (coll. Points politique).

—. La vie de l'esprit. 1 La pensée. Trad. L. Lotringer. Paris : P.U.F., 1981 (coll. Philosophie d'aujourd'hui).

—. La vie de l'esprit. 2 Le vouloir. Trad. L. Lotringer. Paris : P.U.F., 1983 (coll. Philosophie d'aujourd'hui).

Arendt, Hannah et Karl Jaspers. Correspondance (1926-1969). Trad. É. Kaufholz-Messmer. Paris : Payot et Rivages, 1995. 
-. Correspondence, 1926-1969. Dir. L. Kohler et H. Saner (dir.), trad. R. et R. Kimber. New York : Harcourt Brace \& Co., 1992.

Beiner, Ronald et Jennifer Nedelsky (dir.). Judgment, Imagination, and Politics. Themes from Kant and Arendt. Lanham : Rowman \& Littlefield Publishers, 2001.

Bernstein, Richard J. Hannah Arendt and the Jewish Question. Cambridge (MA) : M.I.T. Press, 1996.

—. Radical Evil. A Philosophical Interrogation. Cambridge : Polity Press, 2002.

Collin, Françoise. L'homme est-il devenu superflu? Hannah Arendt. Paris : Odile Jacob, 1999.

Courtine-Denamy, Sylvie. Hannah Arendt. Paris : Hachette Littératures, 1997 (coll. Pluriel).

-. Le souci du monde. Dialogue entre Hannah Arendt et quelques-uns de ses contemporains. Paris : Vrin, 1999 (coll. Pour demain).

Enegrén, André. La pensée politique de Hannah Arendt. Paris : P.U.F., 1984 (coll. Recherches politiques).

Kant, Emmanuel. Critique de la raison pratique. Trad. F. Picavet, Paris : P.U.F., 1966 (coll. Bibliothèque de philosophie contemporaine).

Mathewes, Charles T. Evil and the Augustinian Tradition. Cambridge/New York: Cambridge University Press, 2001.

Neiman, Susan. Evil in Modern Thought. An Alternative History of Philosophy. Princeton/Oxford : Princeton University Press, 2002.

Philonenko, A. L'œuvre de Kant. La philosophie critique. Tome II, Morale et politique. Paris : Vrin, 1997 (coll. À la recherche de la vérité).

Tassin, Étienne. Le trésor perdu. Hannah Arendt l'intelligence de l'action politique, Paris: Payot et Rivages, 1999 (coll. Critique de la politique).

Villa, Dana R. Arendt and Heidegger. The Fate of the Political. Princeton : Princeton University Press, 1996.

Young-Bruehl, Elisabeth. Hannah Arendt. Trad. J. Roman et É. Tassin, Paris : Calmann-Levy, 1999 (coll. Biographie).

—. Why Arendt Matters. New Haven/London : Yale University Press, 2006. 
Young-Bruehl, Elisabeth et Faith Bethelard,. Cherishment. A Psychology of the Heart, New York : The Free Press, 2000.

\section{Documents provenant des Hannah Arendt Papers :}

Arendt, Hannah. Series : Subject File, 1949-1975, The History of the Will, Courses, New School for Social Research, New York, Fall 1971 (1 of 2 folders), The Hannah Arendt Papers, Manuscript Division, Library of Congress, Washington, D.C.

—. Series : Subject File, 1949-1975, Kant's Political Philosophy, Seminar, 1964, 1970, University of Chicago, The Hannah Arendt Papers, Manuscript Division, Library of Congress, Washington, D.C.

Kohn, Jerome. «Evil: The Crime Against Humanity», dans Three Essays: The Role of Experience in Hannah Arendt's Political Thought. The Hannah Arendt Papers, Manuscript Division, Library of Congress, Washington, D.C. 